\title{
Editorial: Small Non-coding RNAs in Streptococci
}

\author{
Mohamed A. Zorgani 1*, Emilie Camiade ${ }^{1}$, Roland Quentin ${ }^{1,2}$ and \\ Marie-Frédérique Lartigue ${ }^{1,2 *}$
}

' ISP, Institut National De La Recherche Agronomique, Université Tours, Equipe Bactéries et Risque Materno-Foetal, UMR 1282, Tours, France, ${ }^{2}$ Centre Hospitalier Régional Universitaire de Tours, Service de Bactériologie Virologie et Hygiène

Hospitalière, Tours, France

Keywords: small RNAs, Streptococcus, cis-acting RNAs, trans-acting RNAs, CRISPR RNAs

\section{The Editorial on the Research Topic}

\section{Small Non-coding RNAs in Streptococci}

Bacterial small RNAs (sRNAs) are post-transcriptional regulators of gene expression and the mechanisms by which this can occur have begun to be understood (Gottesman and Storz, 2011). In pathogenic bacteria, the importance of sRNAs-mediated regulation depends on a fine-tuning of the expression of various virulence genes (Papenfort and Vogel, 2010). For instance, RNAIII, a regulatory RNA from the pathogenic bacterium Staphylococcus aureus, controls the expression

OPEN ACCESS

Edited by:

Stephen Kwok-Wing Tsui,

Chinese University of Hong Kong,

China

Reviewed by:

Cuncong Zhong,

University of Kansas, USA

*Correspondence:

Mohamed A. Zorgani

mohamedamine.zorgani@ etu.univ-tours.fr

Marie-Frédérique Lartigue marie-frederique.lartigue@univ-tours.fr

Specialty section:

This article was submitted to

RNA,

a section of the journal

Frontiers in Genetics

Received: 13 July 2016

Accepted: 17 October 2016

Published: 02 November 2016

Citation:

Zorgani MA, Camiade E, Quentin R and Lartigue M-F (2016) Editorial:

Small Non-coding RNAs in

Streptococci. Front. Genet. 7:192.

doi: 10.3389/fgene.2016.00192 of different virulence factors such as rot (repressor of toxins), spa (surface protein A), sa1000 (fibrinogen binding protein), or coa (coagulase) (Boisset et al., 2007). Considering the functional roles of sRNAs, they are categorized in two major classes: (i) cis-acting elements located/acting on untranslated regions (UTRs) of a translated mRNA which controls the expression of their enclosed gene(s) through the modulation of their secondary structures or stability; and (ii) the trans-acting sRNAs that comprise the cis-encoded sRNAs, known as antisense RNAs (asRNAs), and the transencoded sRNAs which generally present imperfect base-pairing with the mRNA targets (Zorgani et al., 2016).

The goal of this special research topic is to bring together research reviews and original articles on sRNAs, their identification and the characterization of their mode of action in Streptococci. Streptococcal species can colonize and invade humans and animals. They mostly exhibit an asymptomatic interaction within their hosts. However, several of them, such as Streptococcus pyogenes and Streptococcus pneumoniae, are well-known pathogenic species responsible for severe and live-threatening infections in humans (Lamagni et al., 2009). The development of biocomputational and high throughput screening methods, like next generation sequencing, led to the discovery of hundreds of sRNAs encoded by the bacterial genome, which represent 3-5\% of total number of annotated genes regardless of the studied species (Siezen et al., 2010). Currently, the main challenges in the study of these molecules in Streptococci are their identification, as well as the identification of their potential targets. Three review papers published in this research topic reported that a large number of putative regulatory sRNAs were recently identified in different streptococcal species (Cho and Kim; Patenge et al.; Wilton et al.).

Most of these studies focus on three pathogenic species: S. pyogenes and S. pneumoniae and the opportunistic pathogen involved in neonatal infections, Streptococcus agalactiae. At present, 75 sRNAs have been identified in S. pyogenes (Perez et al., 2009; Patenge et al., 2012; Tesorero et al., 2013) and 179 sRNAs in S. pneumoniae (Kumar et al., 2010; Tsui et al., 2010; Acebo et al., 2012; Mann et al., 2012). In S. agalactiae, 197 sRNAs were predicted in silico and 10 of them were validated by northern blot (Pichon et al., 2012). More recently, 125 sRNAs were identified by dRNA-seq (differential RNA-sequencing) in the strain S. agalactiae NEM316 (Rosinski-Chupin et al., 2015). 
Although, several sRNAs have been identified to date in Streptococci, very few are characterized. For instance, in $S$. pyogenes, only three sRNAs were characterized: (i) FasX, which binds to the $5^{\prime}$ end of ska mRNA and activates its translation by avoiding degradation with RNase J1 (Ramirez-Peña et al., 2010); (ii) RivX, which affects the expression of emm, $s c p A, m g a$, and speA mRNAs (Roberts and Scott, 2007); and (iii) Pel, a pleiotropic sRNA which affects the expression of different virulence factors $(\mathrm{emm}, \mathrm{sic}$, and speB) and encodes at the same time an hemolysin (Mangold et al., 2004).

In $S$. pneumoniae, a combination of biocomputational approaches, transcriptomic analysis and RNA-seq were used to identify sRNAs encoded by $S$. pneumoniae. While an in silico study identified 128 sRNAs located in intergenic regions (Livny et al., 2008), 50 sRNAs were identified by microarray analysis (Kumar et al., 2010), and 88 sRNAs by RNA-seq (Acebo et al., 2012). A recent study published in our special research topic demonstrated that csRNAs (cia-dependent small RNAs) are involved in competence control in S. pneumoniae R6 (Laux et al.). The csRNAs are non-coding trans-acting sRNAs transcribed from the promoter associated to CiaRH twocomponent system. They generally present a complementarity to the Shine-Dalgarno sequence and the translation initiation codon (AUG). The authors showed, by using reporter gene fusions, that deletion of a single or two csRNAs was not sufficient to impair competence gene expression. In contrast, combination of three csRNAs, or mutations in the competence gene $\operatorname{com} C$ and its complemented csRNAs, completely blocked S. pneumoniae competence. This study demonstrated that csRNAs are important players in competence regulation for S. pneumoniae.

Two original research articles focused on sRNAs in S. agalactiae were included in the present topic. The first study is about RNAII, an asRNA involved in replication control

\section{REFERENCES}

Acebo, P., Martin-Galiano, A. J., Navarro, S., Zaballos, A., and Amblar, M. (2012). Identification of 88 regulatory small RNAs in the TIGR4 strain of the human pathogen Streptococcus pneumoniae. RNA 18, 530-546. doi: 10.1261/rna.027359.111

Boisset, S., Geissmann, T., Huntzinger, E., Fechter, P., Bendridi, N., Possedko, M., et al. (2007). Staphylococcus aureus RNAIII coordinately represses the synthesis of virulence factors and the transcription regulator Rot by an antisense mechanism. Genes Dev. 21, 1353-1366. doi: 10.1101/gad.423507

Chylinski, K., Le Rhun, A., and Charpentier, E. (2013). The tracrRNA and Cas9 families of type II CRISPR-Cas immunity systems. RNA Biol. 10, 726-737. doi: $10.4161 /$ rna.24321

Gottesman, S., and Storz, G. (2011). Bacterial small RNA regulators: versatile roles and rapidly evolving variations. Cold Spring Harb. Perspect. Biol. 3:a003798. doi: 10.1101/cshperspect.a003798

Kumar, R., Shah, P., Swiatlo, E., Burgess, S. C., Lawrence, M. L., and Nanduri, B. (2010). Identification of novel non-coding small RNAs from Streptococcus pneumoniae TIGR4 using high-resolution genome tiling arrays. BMC Genomics 11:350. doi: 10.1186/1471-2164-11-350

Lamagni, T. L., Neal, S., Keshishian, C., Powell, D., Potz, N., Pebody, R., et al. (2009). Predictors of death after severe Streptococcus pyogenes infection. Emerg. Infect. Dis. 15, 1304-1307. doi: 10.3201/eid1508.090264

Livny, J., Teonadi, H., Livny, M., and Waldor, M. K. (2008). High-throughput, kingdom-wide prediction and annotation of bacterial non-coding RNAs. PLoS ONE 3:e3197. doi: 10.1371/journal.pone.0003197 and encoded by pMV158 plasmid (López-Aguilar et al.). One of the main finding is that RNAII do not requires the formation of a kissing complex for efficient binding to its mRNA target or for inhibition of repB expression. The $\operatorname{rep} B$ gene encodes the replication initiator protein of the pMV158 plasmid. However, site-directed mutagenesis on the $5^{\prime}$ and $3^{\prime}$ regions on the $5^{\prime}$-tail of RNAII, revealed that the entire $5^{\prime}$-tail on the asRNA is mandatory for efficient repB translation in vitro and pMV158 replication in vivo.

The second study described the distribution of the type II-A CRISPR-Cas system among the different $S$. agalactiae genetic lineages (Lier et al.). CRISPR RNAs (Clustered Regularly Interspaced Short Palindromic Repeats RNAs) are sRNAs that target invading cognate nucleic acids (Chylinski et al., 2013). Beside the ubiquitous presence and the high sequence polymorphism of CRISPR1 spacers, one of the main finding of this study is that strains belonging to the "highly virulent" sequence type 17 clone showed a lower number of spacers. This result suggests that sequencing of the CRISPR1 locus can be used as an alternative tool for phylogenetic studies.

The sRNAs widespread in the bacterial kingdom, and particularly in Streptococci, highlights their importance in regard to regulatory networks and pathogenesis and open new perspectives for future therapeutic and industrial applications. This special research topic was the opportunity to review the different sRNAs categories in Streptococci and to emphasize their role in gene regulation, physiology, and virulence.

\section{AUTHOR CONTRIBUTIONS}

MZ: Wrote the manuscript; ML, EC, and RQ: Participated in manuscript correction.

Mangold, M., Siller, M., Roppenser, B., Vlaminckx, B. J., Penfound, T. A., Klein, R., et al. (2004). Synthesis of group A streptococcal virulence factors is controlled by a regulatory RNA molecule. Mol. Microbiol. 53, 1515-1527. doi: 10.1111/j.1365-2958.2004.04222.x

Mann, B., van Opijnen, T., Wang, J., Obert, C., Wang, Y. D., Carter, R., et al. (2012). Control of virulence by small RNAs in Streptococcus pneumoniae. PLoS Pathog. 8:e1002788. doi: 10.1371/journal.ppat.1002788

Papenfort, K., and Vogel, J. (2010). Regulatory RNA in bacterial pathogens. Cell Host Microbe 8, 116-127. doi: 10.1016/j.chom.2010.06.008

Patenge, N., Billion, A., Raasch, P., Normann, J., Wisniewska-Kucper, A., Retey, J., et al. (2012). Identification of novel growth phase- and media-dependent small non-coding RNAs in Streptococcus pyogenes M49 using intergenic tiling arrays. BMC Genomics 13:550. doi: 10.1186/1471-2164-13-550

Perez, N., Treviño, J., Liu, Z., Ho, S. C., Babitzke, P., and Sumby, P. (2009). A genome-wide analysis of small regulatory RNAs in the human pathogen group A Streptococcus. PLoS ONE 4:e7668. doi: 10.1371/journal.pone. 0007668

Pichon, C., du Merle, L., Caliot, M. E., Trieu-Cuot, P., and Le Bouguénec, C. (2012). An in silico model for identification of small RNAs in whole bacterial genomes: characterization of antisense RNAs in pathogenic Escherichia coli and Streptococcus agalactiae strains. Nucleic Acids Res. 40, 2846-2861. doi: 10.1093/nar/gkr1141

Ramirez-Peña, E., Treviño, J., Liu, Z., Perez, N., and Sumby, P. (2010). The group A Streptococcus small regulatory RNA FasX enhances streptokinase activity by increasing the stability of the ska mRNA transcript. Mol. Microbiol. 78, 1332-1347. doi: 10.1111/j.1365-2958.2010.07427.x 
Roberts, S. A., and Scott, J. R. (2007). RivR and the small RNA RivX: the missing links between the CovR regulatory cascade and the Mga regulon. Mol. Microbiol. 66, 1506-1522. doi: 10.1111/j.1365-2958.2007. 06015.x

Rosinski-Chupin, I., Sauvage, E., Sismeiro, O., Villain, A., Da Cunha, V., Caliot, M. E., et al. (2015). Single nucleotide resolution RNA-seq uncovers new regulatory mechanisms in the opportunistic pathogen Streptococcus agalactiae. BMC Genomics 16:419. doi: 10.1186/s12864-015-1583-4

Siezen, R. J., Wilson, G., and Todt, T. (2010). Prokaryotic whole-transcriptome analysis: deep sequencing and tiling arrays. Microb. Biotechnol. 3, 125-130. doi: 10.1111/j.1751-7915.2010.00166.x

Tesorero, R. A., Yu, N., Wright, J. O., Svencionis, J. P., Cheng, Q., Kim, J. H., et al. (2013). Novel regulatory small RNAs in Streptococcus pyogenes. PLoS ONE 8:e64021. doi: 10.1371/journal.pone.0064021

Tsui, H. C., Mukherjee, D., Ray, V. A., Sham, L. T., Feig, A. L., and Winkler, M. E. (2010). Identification and characterization of noncoding small RNAs in
Streptococcus pneumoniae serotype 2 strain D39. J. Bacteriol. 192, 264-279. doi: 10.1128/JB.01204-09

Zorgani, M. A., Quentin, R., and Lartigue, M. F. (2016). Regulatory RNAs in the less studied streptococcal species: from nomenclature to identification. Front. Microbiol. 7:1161. doi: 10.3389/fmicb.2016.01161

Conflict of Interest Statement: The authors declare that the research was conducted in the absence of any commercial or financial relationships that could be construed as a potential conflict of interest.

Copyright (c) 2016 Zorgani, Camiade, Quentin and Lartigue. This is an open-access article distributed under the terms of the Creative Commons Attribution License (CC $B Y)$. The use, distribution or reproduction in other forums is permitted, provided the original author(s) or licensor are credited and that the original publication in this journal is cited, in accordance with accepted academic practice. No use, distribution or reproduction is permitted which does not comply with these terms. 